\title{
Liver transplantation for alcoholic hepatitis
}

\author{
Lindsey C. Shipley ${ }^{1}$, Ashwani K. Singal ${ }^{2}$ \\ ${ }^{1}$ Division of Internal Medicine, University of Alabama, Birmingham, AL, USA; ${ }^{2}$ Division of Gastroenterology and Hepatology, University of South \\ Dakota, Avera McKennan University Health Center and Transplant Institute, Sioux Falls, SD, USA \\ Contributions: (I) Conception and design: AK Singal; (II) Administrative support: AK Singal; (III) Provision of study materials or patients: None; \\ (IV) Collection and assembly of data: L Shipley, AK Singal; (V) Data analysis and interpretation: L Shipley, AK Singal; (VI) Manuscript writing: All \\ Authors; (VII) Final approval of manuscript: All Authors. \\ Correspondence to: Ashwani K. Singal, MD, MS, FACG, FAASLD. Associate Professor of Medicine, Division of Gastroenterology and Hepatology, \\ University of South Dakota, Transplant Hepatologist and Chief Clinical Research Affairs, Avera University Hospital, Transplant Institute, and \\ Institute of Human Genetics Research, Sioux Falls, SD 57105, USA. Email: ashwanisingal.com@gmail.com.
}

\begin{abstract}
Alcoholic hepatitis $(\mathrm{AH})$ is associated with a high short-term mortality. Currently, most transplant centers require minimum six months of abstinence from alcohol use before considering liver transplant for patients with end stage liver disease. Some recent data are emerging on the benefits and safety of early liver transplantation for patients with severe $\mathrm{AH}$, a population who cannot meet the minimum six months sobriety. This article reviews the current status, benefits, challenges, barriers, and future prospects on early liver transplantation in patients with severe, acute $\mathrm{AH}$.
\end{abstract}

Keywords: Alcoholic liver disease; liver transplantation; alcohol use post-transplant; allograft survival

Received: 03 July 2019; Accepted: 05 November 2019; Published: 05 April 2020.

doi: $10.21037 /$ tgh.2019.11.17

View this article at: http://dx.doi.org/10.21037/tgh.2019.11.17

\section{Introduction}

Alcoholic hepatitis $(\mathrm{AH})$ is a distinct clinical syndrome among individuals with chronic and active harmful alcohol consumption, 60 days of heavy consumption ( $>50 \mathrm{~g} /$ day) of alcohol for a minimum of six months. Patients present with jaundice, a serum bilirubin $>3 \mathrm{mg} / \mathrm{dL}$, an elevated AST (50-400 U/L), an AST:ALT ratio $>1.5$ and no other obvious cause for hepatitis (1). Currently, there is no role for liver biopsy prior to transplant unless there is clinical diagnostic doubt or concern for dual pathology (2). Further, $\mathrm{AH}$ is associated with a high mortality, chance of death, of up to $30 \%$ over a period of 1 month from presentation (3-6). The cornerstone treatment for $\mathrm{AH}$ includes abstinence and nutrition. Steroids are the only pharmacological option in severe $\mathrm{AH}$ and are recommended by AASLD, EASL, and ACG as first line treatment for patients with severe $\mathrm{AH}(3,7)$. However, these drugs are limited by potential for adverse effects, unpredictable benefit in $40-60 \%$ cases, and survival benefit for only 1 month from presentation $(8-10)$. This is further compounded by heterogeneity on use of these drugs by physicians in routine clinical practice among severe $\mathrm{AH}$ patients who are otherwise eligible for corticosteroid therapy (8). Clearly, there is an unmet clinical need for newer therapies for patients with severe AH. Many therapeutic targets are under diligent investigation in NIAAA funded clinical trials. But, currently none of these therapies are FDA approved and available to physicians in their practices (11). In the background of emerging data on early liver transplantation (LT) in severe AH patients, this article attempts to review the current status of LT in AH and highlight the current challenges, barriers, and future prospects on this therapy for these sick patients with severe AH.

\section{Rationale for liver transplant in AH}

In the background of high short-term mortality and lack of available effective medical therapies, LT becomes a salvage option for these patients, and has a strong clinical rationale and premise. However, as these patients are drinking often until or right before their presentation 
and transplant centers universally require minimum six months of abstinence from alcohol consumption before even considering for evaluation, LT for severe AH has remained controversial. Further, in view of shortage of organs combined with concern for relapse and the public perception that this is a self-inflicted disease have been ongoing challenges to consider LT as treatment option for patients with severe $\mathrm{AH}$ (6).

\section{Six months rule}

The rationale behind the six-month abstinence requirement was to allow the liver to improve and regenerate and then consider transplant if this period of sobriety has not resulted in improvement in liver function and/or decreased episodes of decompensation. However, since then the six months criterion is used as a rule by transplant centers as a requirement for transplant evaluation with the fear that less than six months of sobriety is a risk factor for return to alcohol use after transplantation $(12,13)$. Of the various predictors of relapse to alcohol use after transplant, six months abstinence rule seems a weak predictor with most important predictors being patient's psychosocial status, younger age, and family history of alcoholism $(6,14)$. For example, in a systematic review of over twenty studies examining predictors of alcohol use after liver transplantation, six months abstinence came out as a predictor in only 2 of the nine studies. In contrast, psychosocial status of the candidate predicted in six of seven studies, young age in two of three studies, and family history of alcoholism in three of four studies. When comparing to those with AAH and bland alcoholic cirrhosis (BAC), posttransplant patient and graft survival between $\mathrm{AAH}$ and BAC were similar ( $\mathrm{P}=0.13, \mathrm{P}=0.11$, respectively) and there was no differences in relapse $(\mathrm{P}=0.13)(15)$. Other strong predictors in this analysis were current polysubstance abuse and alcohol rehabilitation treatment failures (16). Further, a study by Dom et al., showed longer periods of abstinence from alcohol beyond six months can have a stronger prognostic value flor low risk of post-transplant relapse at $5 \%$ per month (17). However, some insurance companies still require the 6-month rule (18).

\section{Emerging data}

The possibility of considering liver transplantation in AH patients dates back to as early as 1995 (Table 1). In a retrospective study, 209 patients with alcohol associated liver disease including patients with $\mathrm{AH}$, acute and chronic rejection episodes were assessed based on relapse to alcohol use. The authors showed graft rejection rate over a median period of over 4 years to be higher among abstinent compared to those who relapsed to alcohol use (0.75 episodes/patient) (2.24 vs. 0.75 episodes/year, $\mathrm{P}<0.01)$. Similar data was reported for comparing chronic rejection rates which occurred in $26(12.4 \%)$ patients (19). In another retrospective study on nine patients with severe $\mathrm{AH}$ $(\mathrm{mDF}>32)$ and unreliable alcohol history, treatment response to graft rejection and survival was similar irrespective of abstinence post-transplant.

Since then three retrospective studies have examined explants among recipients for alcohol associated liver disease for changes of $\mathrm{AH}$ and assessed survival of these patients to those with bland cirrhosis (Table 1). Of 110, 148, and 62 transplants for alcohol associated liver disease 8, 32, and 36 respectively had changes of $\mathrm{AH}$ in the explants. The overall survival of patients with $\mathrm{AH}$ changes on explants was similar compared to patients with bland cirrhosis in all these three studies $(20,21)$. When compared to alcoholic liver disease, patient and graft survival was not significantly different $(\mathrm{P}=0.53)$ and had no difference in post-transplant relapse $(\mathrm{P}=0.13)(15)$. In one of these studies, the rates on post-transplant relapse to alcohol use were also similar, $\mathrm{P}=0.13$ (15).

In a landmark case control prospective study, the 'six months rule' was challenged by the French workers. In this study, select 26 patients with severe AH who did not respond to steroid therapy received a LT. The six months survival of these patients as compared to matched 26 severe $\mathrm{AH}$ patients who were not selected to receive liver transplant was significantly improved (77\% vs. $23 \%$, $\mathrm{P}<0.001)$. Only one of the three patients who self-reported relapse to alcohol use reported heavy alcohol use at a two year follow up (13).

Since this pivotal study, there has been lot of enthusiasm in this field and many prospective case series, retrospective and database studies, survey of transplant centers, and multicenter consortium data have emerged supporting the beneficial role of early liver transplantation in severe $\mathrm{AH}$ patients (Table 1) (29). For example, in an analysis of the UNOS dataset comparing alcoholic cirrhosis versus $\mathrm{AH}$, the five-year graft survival was $73 \%$ and $75 \%(\mathrm{P}=0.97)$ and five-year patient survival, $78 \%$ and $80 \%(\mathrm{P}=0.90)$, respectively (23). Additionally, a few retrospective studies 
Table 1 Studies to date examining liver transplant in alcoholic hepatitis

\begin{tabular}{|c|c|c|c|c|c|}
\hline Author/year & Location of study & Study design & Sample size $(\mathrm{AH})$ & Primary outcomes & 6 mo. survival \\
\hline Shakil et al., 1997 (20) & USA & Retrospective & 9 & $\begin{array}{l}\text { Graft survival and relapse } \\
\text { after LT }\end{array}$ & $89 \%$ \\
\hline Wells et al., 2007 (15) & USA & Retrospective & 32 & $\begin{array}{l}\text { Graft survival and relapse } \\
\text { after } L T\end{array}$ & $96 \%$ \\
\hline Immordino et al., 2009 (21) & France & Retrospective & 8 & Relapse after LT & N/A \\
\hline Mathurin et al., 2011 (13) & France & Prospective & 26 & Graft survival & $77 \%$ \\
\hline Tomé et al., 2002 (22) & Spain & Retrospective & 36 & $\begin{array}{l}\text { Graft survival and relapse } \\
\text { after } L T\end{array}$ & $79 \%$ \\
\hline Im et al., 2016 (24) & USA & Retrospective & 16 & Graft survival and relapse & $88 \%$ \\
\hline Lee et al., 2017 (25) & USA & Retrospective & 17 & $\begin{array}{l}\text { Graft survival and relapse } \\
\text { after LT }\end{array}$ & $100 \%$ \\
\hline Lee et al., 2018 (26) & USA & Retrospective & 147 & Mortality and relapse & $94 \%$ (1 year) \\
\hline Bangaru et al., 2018 (27) & USA & Retrospective & $\begin{array}{l}23 \text { performed } \mathrm{LT} \\
\text { for } \mathrm{AH}\end{array}$ & $\begin{array}{l}\text { Selection criteria } \\
\text { consensus and graft } \\
\text { survival }\end{array}$ & $73.9 \%$ \\
\hline Lee et al., 2019 (28) & USA & Retrospective & 147 & $\begin{array}{l}\text { Patient survival and relapse } \\
\text { to alcohol use }\end{array}$ & $\mathrm{N} / \mathrm{A}$ \\
\hline
\end{tabular}

$\mathrm{AH}$, alcoholic hepatitis; LT, liver transplantation; ALD, alcohol-associated liver disease.

examining transplant explants showed no difference in graft survival when comparing BAC versus AH $(15,30)$. Data from surveys of transplant centers in the United States also showed similar results with graft survival at $73.9 \%$, $88 \%$ and $100 \%$ at 6 months at $94 \%$ at 1 year in one study among patients receiving liver for a diagnosis of severe $\mathrm{AH}$ $(18,25-27)$. In a recently reported retrospective study from the US "Accelerate Consortium", the patient survival among select $147 \mathrm{AH}$ patients receiving early LT without meeting six months abstinence criterion was $94 \%$ at one and $84 \%$ at 3 years. The relapse to sustained alcohol use of $10 \%$ at 1 and $17 \%$ at 3 years negatively impacted outcomes (28).

In a meta-analysis of 11 studies evaluating the role of early liver transplant in $\mathrm{AH}$, the authors showed one-year survival of $85 \%$ with no difference when compared to patients transplanted for alcoholic cirrhosis with six months of abstinence, and over 16 folds survival benefit compared to $\mathrm{AH}$ patients who did not receive liver transplant. Further, the relapse to alcohol consumption in this analysis was $14 \%$ at two years, similar to what is observed in patients transplanted for alcoholic cirrhosis (14). In another metaanalysis of studies examining risk of relapse to alcohol use after liver transplantation, pooled risk was $5-6 \%$ per 100 person years for any and $2-3 \%$ for harmful alcohol use (31). However, a difference in alcohol rates in studies may be attributed to accurate reporting or monitoring and how the individual study measures relapse (drinks versus sustained or harmful use).

Based on this encouraging data, American College of Gastroenterology (ACG) and European Association for the Study of the Liver (EASL) recommend to consider early LT as a salvage therapy for select patients with severe $\mathrm{AH}(3,32)$. Selection criteria need optimization with uniform protocol across LT centers all over the world as basis for using early 
Table 2 Selection criteria for liver transplant in alcoholic hepatitis (13)

- First episode of hepatic decompensation and alcoholic hepatitis

- Severe AH non-responsive to corticosteroid therapy

- Excellent psychosocial status as agreed upon by everyone in the primary treating team (nurse, social worker, addiction team, surgeon and anesthesiologist)

- $\quad$ Signed document by recipient for alcohol rehabilitation, if indicated

$\mathrm{AH}$, alcoholic hepatitis.

LT for patients with severe AH.

\section{Selection criteria}

One of the most debatable issues with LT in AH is proper selection of patients who should be transplanted: those who are likely to die without a LT and those who are likely to maintain abstinence. About $25 \%$ of those nonresponsive to steroid therapy will recover with medical management alone, and if transplanted early, would divert a liver that could have otherwise been used for an alternative candidate (33). There is concern that use of organs for severe acute $\mathrm{AH}$, would divert organs away from other patients. However, based on selection criteria, only $2-3 \%$ would be eligible for LT $(13,18)$.

Risk of relapse to alcohol use after transplant for patients with alcohol associated liver disease is a genuine concern as long-term outcome of patients who engage in harmful alcohol use after transplantation is reduced compared to patients who remain abstinent. Further, the graft remains at risk for $\mathrm{AH}$ and alcohol associated chronic damage including recurrent alcoholic cirrhosis. Clearly, considering patients with severe $\mathrm{AH}$ at high risk for relapse to alcohol use remains a challenge as basis for developing validated protocol to select right patients with acceptable risk for relapse to alcohol use. There have been no prognostic scores validated specifically in the early liver transplant for $\mathrm{AH}$ population, to date, however three have been developed for clinical practice. In a recent retrospective study, the sustained alcohol uses post-LT (SALT) score used four objective pre-transplant variables to identify candidates for early LT who are at low risk for relapse to alcohol use posttransplant. The variables used to identify patients at high risk included (I) $>10$ drinks/day at initial hospitalization (4 points), (II) multiple prior rehabilitation attempts (4 points), (III) prior alcohol-related legal issues (2 points), (IV) prior illicit substance abuse (1 point). Those with a score of $<5$ had a $95 \%$ negative predictive value for sustained alcohol use post-LT (95\% CI: 89-98\%) (34). However, more data is needed to address external validation. Further, the score has a low positive predictive value and thus, a high SALT should not prevent considerations of early liver transplant. Stanford Integrated Psychosocial Assessment for Transplant (SIPAT) is another scoring system to assess a patient's readiness level and illness management. The variables used to identify patients at high risk include (I) knowledge and understanding of medical illness process (4 points), (II) knowledge and understanding of the process of transplantation (4 points), (III) willingness/desire for treatment (transplant) (4 points), (IV) history of treatment adherence/compliance (8 points) and (V) lifestyle factors (4 points). One prospective study suggested higher pre-LT scores associated with increased psychosocial outcomes, hospitalizations and rejection episodes $(35,36)$. High-Risk Alcoholism Relapse Scale (HRAR) assesses duration of heavy drinking, number of drinks per day and number of prior alcoholism inpatient treatment experiences. Duration of heavy drinking $<11$ years gives 0 points, $11-25$ ( 1 point) and $>25$ years ( 2 points). Number of drinks per day is separated into <9 (0 points), 9-17 (1 point) and $>17$ (2 points) and number of prior inpatient treatment experiences of 0 (0 points), 1 ( 1 point) and $>1$ ( 2 points)..One study showed a score $>4$ was associated with increased risk for relapse post-LT however, another study did no show an association (36-38).

Severe $\mathrm{AH}$ is characterized an acute and rapid decompensation within 60 days of heavy consumption for a minimum of six months without another cause who develops multi-organ failure syndrome, including renal impairment (1). Early identification of these patients with a high likelihood for success after liver transplant is important. Maddrey's discriminant function (mDF), MELD and Lille model have good negative predictive values (NPV). A 30- and 90-day mortality NPV for $\mathrm{mDF}$ is 1.00 and 1.00, MELD has 0.96 and 0.96 and Lille 0.91 and 0.86 , respectively. This study also had cut-off scores for each 
Table 3 Barriers for transplant centers on liver transplant in alcoholic hepatitis (18)

- Lack of center protocol

- Socio-cultural factors of the center and community

- Concern for relapse and poor compliance

- Selection of right candidates especially evaluation of psychosocial support

- Public perceptions with organ shortage

- Insurance approval

- No obvious barriers but center policy for not transplanting $\mathrm{AH}$ patients

$\mathrm{AH}$, alcoholic hepatitis.

prognostic model of 32, 21 and 0.45 , respectively (39). Optimal criteria for selection (Table 2) (13) will better optimize use of the donor pool and improve mortality in eligible candidates $(33,40)$. Current criteria for patient selection are based on the European prospective study and include: (I) severe AH non-responsive to corticosteroid therapy predicted by the Lille model score of $<0.45$, (II) excellent psychosocial status as agreed upon by everyone in the treating team including primary team with nurse, social worker with deaddiction team, surgeon and anesthesiologist, (III) first episode of hepatic decompensation especially of AH, and (IV) signed document by the candidate to maintain abstinence after LT and agreement for rehabilitation therapy if recommended by treating team (13). Based on this selection criteria, only approximately $2-3 \%$ of patients would be eligible for LT with no major impact on the donor pool $(13,18)$. On the other end of spectrum, it may not be appropriate to consider LT in patients where further treatment is futile, such as patients being treated in the ICU with $\geq 4$ organ failures or CLIF-ACLF score of $>/=70$. This care becomes futile because any further interventions would be incapable of producing any useful result. However, in those eligible for LT, the discussion should start on arrival to the intensive care unit as the time to death and the window for intervention such as LT is short (41).

The aim of bridging devices is to stabilize a patient until transplant or recovery. Bridging devices fall into one of two categories: liver support devices (biological and nonbiological) and hepatocyte transplantation. Extracorporeal liver-support systems have shown a good safety profile, however have not shown major improvements in synthetic function. There was more improvement in the liver transplant groups versus recovery group however, this data was skewed by those who received transplantation (42). Further, in a recent randomized clinical trial these devices were shown to not improve outcomes. Hepatocyte transplantation with liver progenitor cells is another emerging bridging therapy under study however, current studies lacks data on long-term safety and effectiveness however, appears promising (43).

Properly selected patients with severe AH constitute only $2-3 \%$ of the whole pool of AH patients $(13,23,24)$. Given the magnitude of severe $\mathrm{AH}$ in the general population and the existing donor pool, a continuing debate remains the impact of strategy of transplanting for severe $\mathrm{AH}$ patients on the existing donor pool (23). Larger multicenter prospective studies are needed to examine transplant data in patients with $\mathrm{AH}$ in order to develop uniform protocols for patient selection and scoring systems for graft allocation.

\section{Barriers and future prospects}

As per National Inpatient Database estimates, about 55,000 patients are admitted each year in the US primary or secondary discharge diagnosis of $\mathrm{AH}$, and $20 \%$ of these patients have severe AH and may qualify for LT (44). The majority of physician and center based barriers are related to awareness and liver transplant center protocols (Table 3) (18). However, most transplant centers are interested and are considering liver transplant for patients who present with severe AH (18). It is important that these centers create a formal center protocol to guide patient selection and posttransplant follow-up.

In the previously described pivotal French study, the six-month survival after liver transplant was $77 \%$ vs. $23 \%$ $(\mathrm{P}<0.001)$ and maintained through 2 years of follow-up $(\mathrm{P}<0.004)$ with five of the six deaths related to invasive fungal infections within 2 weeks of transplant. This data represents a potential learning curve for transplant centers as the current rate of one-year survival after LT is over $95 \%$. This study also stresses the importance of removing the 6-month rule as the majority of patients in this study died within 1 month (13). Another study suggested if the patient is able to sustain abstinence after LT, the oneyear survival rate is $94 \%$ [ $95 \%$ confidence interval (CI), $89-97 \%$ ] and three-year survival rate $84 \%$ (95\% CI, $75-90 \%)$ (26). Another barrier is the concern that willingness to donate will be affected if transplant guidelines are mended to include $\mathrm{AH}$. However, this has not been the case for intravenous drug users with hepatitis $\mathrm{B}$ or $\mathrm{C}$ virus or 
self-induced acetaminophen overdose (45). Many transplant centers agree with addiction rehabilitation, however less than half of centers offer support therapy or encourage patients to attend support groups after LT for AH (46). However, the public perception can complicate the issue further. Results from a recent survey of the public suggest that the majority $(81.5 \%, \mathrm{n}=410)$ were neutral toward early LT in AAH (47). There is an unmet clinical need to develop strategies in developing multidisciplinary teams involving addiction medicine and psychologists as basis for following these patients and prevent alcohol relapse in most highrisk candidates. Additionally, collaboration with research specialists in addiction medicine will be helpful to optimize therapy for these patients. It will also be important to understand the impact of LT for AH on clinical workload and cost at the center level and may help guide transplant center protocols $(17,48)$.

\section{Conclusions}

Early liver transplant for AAH previously had not been considered as an indication. The general consensus was concern for relapse, shortage of organs and the idea that the disease is self-induced. Emerging data suggest that current guidelines for early LT in this population such as the 6-month abstinence rule do not change rates for relapse to alcohol or survival suggesting that thousands of eligible patients are currently not being considered for a potential lifesaving procedure. Future prospects include creating (I) a formal protocol to select eligible patients with $\mathrm{AH}$ for liver transplantation, (II) guidelines for appropriate followup, (III) addiction medicine treatment guidelines and (IV) better immunosuppression regimens, especially against invasive fungal organisms. Larger multicenter prospective studies are needed to examine transplant data survival and outcomes in patients with $\mathrm{AH}$.

\section{Acknowledgments}

None.

\section{Footnote}

Conflicts of Interest: The authors have no conflicts of interest to declare.

Ethical Statement: The authors are accountable for all aspects of the work in ensuring that questions related to the accuracy or integrity of any part of the work are appropriately investigated and resolved.

\section{References}

1. Mitchell MC, Friedman LS, McClain CJ. Medical Management of Severe Alcoholic Hepatitis: Expert Review from the Clinical Practice Updates Committee of the AGA Institute. Clin Gastroenterol Hepatol 2017;15:5-12.

2. Dhanda AD, Collins PL, McCune CA. Is liver biopsy necessary in the management of alcoholic hepatitis? World J Gastroenterol 2013;19:7825-9.

3. Singal AK, Bataller R, Ahn J, et al. ACG Clinical Guideline: Alcoholic Liver Disease. Am J Gastroenterol 2018;113:175-94.

4. Singal AK, Kodali S, Vucovich LA, et al. Diagnosis and Treatment of Alcoholic Hepatitis: A Systematic Review. Alcohol Clin Exp Res 2016;40:1390-402.

5. Singal AK, Louvet A, Shah VH, et al. Grand Rounds: Alcoholic Hepatitis. J Hepatol 2018;69:534-43.

6. O'Shea RS, Dasarathy S, McCullough AJ, et al. Alcoholic liver disease. Hepatology 2010;51:307-28.

7. Thursz M, Kamath PS, Mathurin P, et al. Alcoholrelated liver disease: Areas of consensus, unmet needs and opportunities for further study. J Hepatol 2019;70:521-30.

8. Singal AK, Salameh H, Singal A, et al. Management practices of hepatitis $\mathrm{C}$ virus infected alcoholic hepatitis patients: A survey of physicians. World J Gastrointest Pharmacol Ther 2013;4:16-22.

9. Singal AK, Shah VH, Kamath PS. Infection in Severe Alcoholic Hepatitis: Yet Another Piece in the Puzzle. Gastroenterology 2017;152:938-40.

10. Singal AK, Shah VH. Therapeutic Strategies for the Treatment of Alcoholic Hepatitis. Semin Liver Dis 2016;36:56-68.

11. Singal AK, Shah VH. Current trials and novel therapeutic targets for alcoholic hepatitis. J Hepatol 2019;70:305-13.

12. Rice JP, Lucey MR. Should length of sobriety be a major determinant in liver transplant selection? Curr Opin Organ Transplant 2013;18:259-64.

13. Mathurin P, Moreno C, Samuel D, et al. Early liver transplantation for severe alcoholic hepatitis. $\mathrm{N}$ Engl J Med 2011;365:1790-800.

14. Marot A, Dubois M, Trepo E, et al. Liver transplantation for alcoholic hepatitis: A systematic review with metaanalysis. PLoS One 2018;13:e190823.

15. Wells JT, Said A, Agni R, et al. The impact of acute alcoholic hepatitis in the explanted recipient liver on 
outcome after liver transplantation. Liver Transpl 2007;13:1728-35.

16. McCallum S, Masterton G. Liver transplantation for alcoholic liver disease: a systematic review of psychosocial selection criteria. Alcohol Alcohol 2006;41:358-63.

17. Dom G, Peuskens H. Addiction specialist's role in liver transplantation procedures for alcoholic liver disease. World J Hepatol 2015;7:2091-9.

18. Hasanin M, Dubay DA, McGuire BM, et al. Liver transplantation for alcoholic hepatitis: A survey of liver transplant centers. Liver Transpl 2015;21:1449-52.

19. Van Thiel DH, Bonet H, Gavaler J, et al. Effect of alcohol use on allograft rejection rates after liver transplantation for alcoholic liver disease. Alcohol Clin Exp Res 1995;19:1151-5.

20. Shakil AO, Pinna A, Demetris J, et al. Survival and quality of life after liver transplantation for acute alcoholic hepatitis. Liver Transpl Surg 1997;3:240-4.

21. Immordino G, Gelli M, Ferrante R, et al. Alcohol abstinence and orthotopic liver transplantation in alcoholic liver cirrhosis. Transplant Proc 2009;41:1253-5.

22. Tomé S, Martinez-Rey C, González-Quintela A, et al. Influence of superimposed alcoholic hepatitis on the outcome of liver transplantation for end-stage alcoholic liver disease. J Hepatol 2002;36:793-8.

23. Singal AK, Bashar H, Anand BS, et al. Outcomes after liver transplantation for alcoholic hepatitis are similar to alcoholic cirrhosis: exploratory analysis from the UNOS database. Hepatology 2012;55:1398-405.

24. Im GY, Kim-Schluger L, Shenoy A, et al. Early Liver Transplantation for Severe Alcoholic Hepatitis in the United States--A Single-Center Experience. Am J Transplant 2016;16:841-9.

25. Lee BP, Chen PH, Haugen C, et al. Three-year Results of a Pilot Program in Early Liver Transplantation for Severe Alcoholic Hepatitis. Ann Surg 2017;265:20-9.

26. Lee BP, Mehta N, Platt L, et al. Outcomes of Early Liver Transplantation for Patients With Severe Alcoholic Hepatitis. Gastroenterology 2018;155:422-430.e1.

27. Bangaru S, Pedersen MR, MacConmara MP, et al. Survey of Liver Transplantation Practices for Severe Acute Alcoholic Hepatitis. Liver Transpl 2018;24:1357-62.

28. Lee BP, Mehta N, Platt L, et al. Outcomes of Early Liver Transplantation for Patients With Severe Alcoholic Hepatitis. Gastroenterology 2018;155:422-30.e1.

29. Mackie J, Groves K, Hoyle A, et al. Orthotopic liver transplantation for alcoholic liver disease: a retrospective analysis of survival, recidivism, and risk factors predisposing to recidivism. Liver Transpl 2001;7:418-27.

30. Tome S, Martinez-Rey C, Gonzalez-Quintela A, et al. Influence of superimposed alcoholic hepatitis on the outcome of liver transplantation for end-stage alcoholic liver disease. J Hepatol 2002;36:793-8.

31. Dew MA, DiMartini AF, Steel J, et al. Meta-analysis of risk for relapse to substance use after transplantation of the liver or other solid organs. Liver Transpl 2008;14:159-72.

32. easloffice@easloffice.eu EAftSotLEa, Liver EAftSot. EASL Clinical Practice Guidelines: Management of alcoholrelated liver disease. J Hepatol 2018;69:154-81.

33. Daswani R, Kumar A, Sharma P, et al. Role of liver transplantation in severe alcoholic hepatitis. Clin Mol Hepatol 2018;24:43-50.

34. Lee BP, Vittinghoff E, Hsu C, et al. Predicting Low Risk for Sustained Alcohol Use After Early Liver Transplant for Acute Alcoholic Hepatitis: The Sustained Alcohol Use Post-Liver Transplant Score. Hepatology 2019;69:1477-87.

35. Maldonado JR, Sher Y, Lolak S, et al. The Stanford Integrated Psychosocial Assessment for Transplantation: A Prospective Study of Medical and Psychosocial Outcomes. Psychosom Med 2015;77:1018-30.

36. Lim J, Sundaram V. Risk factors, scoring systems, and interventions for alcohol relapse after liver transplantation for alcoholic liver disease. Clin Liver Dis (Hoboken) 2018;11:105-10.

37. De Gottardi A, Spahr L, Gelez P, et al. A simple score for predicting alcohol relapse after liver transplantation: results from 387 patients over 15 years. Arch Intern Med 2007;167:1183-8.

38. DiMartini A, Day N, Dew MA, et al. Alcohol consumption patterns and predictors of use following liver transplantation for alcoholic liver disease. Liver Transpl 2006;12:813-20.

39. Lee BP, Terrault NA. Early liver transplantation for severe alcoholic hepatitis: moving from controversy to consensus. Curr Opin Organ Transplant 2018;23:229-36.

40. Shah VH. Managing alcoholic liver disease. Clin Mol Hepatol 2015;21:212-9.

41. Engelmann C, Thomsen KL, Zakeri N, et al. Validation of CLIF-C ACLF score to define a threshold for futility of intensive care support for patients with acute-on-chronic liver failure. Crit Care 2018;22:254.

42. Singhal A, Neuberger J. Acute liver failure: bridging to transplant or recovery--are we there yet? J Hepatol 2007;46:557-64.

43. Nyberg SL. Bridging the gap: advances in artificial liver 
support. Liver Transpl 2012;18 Suppl 2:S10-4.

44. Singal AK, Kuo YF, Anand BS. Hepatitis C virus infection in alcoholic hepatitis: prevalence patterns and impact on in-hospital mortality. Eur J Gastroenterol Hepatol 2012;24:1178-84.

45. Singal AK, Chaha KS, Rasheed K, et al. Liver transplantation in alcoholic liver disease current status and controversies. World J Gastroenterol 2013;19:5953-63.

46. Weeks SR, Sun Z, McCaul ME, et al. Liver

doi: $10.21037 /$ tgh.2019.11.17

Cite this article as: Shipley LC, Singal AK. Liver transplantation for alcoholic hepatitis. Transl Gastroenterol Hepatol 2020;5:26.
Transplantation for Severe Alcoholic Hepatitis, Updated Lessons from the World's Largest Series. J Am Coll Surg 2018;226:549-57.

47. Stroh G, Rosell T, Dong F, et al. Early liver transplantation for patients with acute alcoholic hepatitis: public views and the effects on organ donation. Am J Transplant 2015;15:1598-604.

48. Im GY, Cameron AM, Lucey MR. Liver transplantation for alcoholic hepatitis. J Hepatol 2019;70:328-34. 